\title{
Performances of Erbium-Doped Fiber Amplifier at 980-nm Pump Power in Various Parameter Combinations
}

\author{
Masudur Rahman Siddiquee ${ }^{1}$, Naznin Akter $^{1} \&$ Sazzad Muhammad Samaun Imran ${ }^{1}$ \\ ${ }^{1}$ Department of Applied Physics, Electronics \& Communication Engineering, University of Dhaka, Dhaka-1000, \\ Bangladesh \\ Correspondence: Masudur Rahman Siddiquee, Department of Applied Physics, Electronics \& Communication \\ Engineering, University of Dhaka, Bangladesh. Tel: 88-017-1194-8327. E-mail: masud518@gmail.com
}

Received: May 28, 2014 Accepted: August 6, 2015 Online Published: August 14, 2015

doi:10.5539/apr.v7n5p60 URL: http://dx.doi.org/10.5539/apr.v7n5p60

\begin{abstract}
Optical fiber amplifiers are dominating the loss compensation in fiber optic communications systems, especially the Erbium doped types for communication spectrum based on silica fibers. The gain of the EDFA can be maximized by using appropriate design parameters and appropriate value of the material properties. This paper describes the derivation of the equations that govern the pump power, input signal propagation, gain and optimum fiber length of the amplifier. By using these deduced expressions, new tools have been developed using $\mathrm{C}$ programming and MATLAB and then the performances of EDFA pumped with 980-nm signal have been analyzed. The analysis results that the gain of the amplifier increases with the length of the amplifier, and also with the pump signal power. At higher values of signal power the gain becomes saturated. The gain has a nonlinear relation with the amplifier length for a particular pump power. From the experiments, it has been found that for a particular length the saturation gain increases with the carrier density of the amplifier, but at longer length the gain is almost independent of the carrier density. It has also been found that the pump threshold power increases linearly with the length and carrier density of the amplifier. The tools developed in the analysis process could be used by researchers in future for further investigation of the performance of EDFA in various parameter sets or, for finding a parameter set for specific target performance of the EDFA.
\end{abstract}

Keywords: fiber amplifier, erbium-doped fiber amplifier, rate equations, pump threshold power, gain

\section{Introduction}

Optical fibers act as the gain media for Fiber Amplifiers. In most cases, the gain medium is a glass fiber doped with rare earth ions such as erbium, neodymium, ytterbium, praseodymium, or thulium. Erbium-doped fiber amplifiers (EDFA) are the by far most important fiber amplifiers in the context of long-range optical fiber communications. They can efficiently amplify light in the $1.5 \mu \mathrm{m}$ wavelength region, where telecom fibers have their loss minimum (Mears, Reekie, Jauncey, \& Payne, 1987).

The main components of an EDFA should be at least consists of:

1) Erbium-doped optical fiber,

2) Pump laser, and

3) Wavelength-selective coupler.

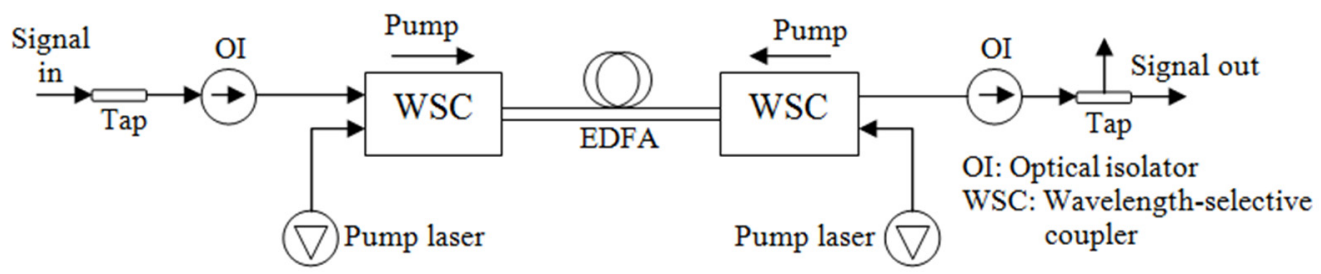

Figure 1. Dual pumping configuration of an EDFA (Mears, Reekie, Jauncey, \& Payne, 1987) 
Pump light is guided into the erbium-doped fiber by means of wavelength division multiplexing (WDM), which is used to couple the pump signal into the doped fiber. Additionally, an isolator is generally placed at the output of an amplifier to prevent back reflection which can degrade amplifier performance or cripple the amplifier due to laser oscillation in the amplifier. Typically, the EDFA configuration can be characterized by pumping schemes into three particular arrangements. These schemes are:

1) Forward-pumped (co-pumped),

2) Backward-pumped (counter-pumped),

3) Dual-pumped.

Efficient EDFA pumping is possible using semiconductor lasers operating near 980- and 1480-nm wavelengths. The required pump power can be reduced by silica fibers doped with aluminum and phosphorus or by using fluorophosphates fibers with the availability of visible semiconductor lasers. EDFAs can also be pumped in the wavelength range 0.6-0.7 $\mu \mathrm{m}$ (Khare, 2004).

An EDFA is a vital building block in many of the long-haul lightwave systems. The EDFA characteristics such as gain, saturation power and spontaneous amplifier noise play a key role in determining the performance of lightwave link. In theory, the gain and saturation characteristics of an EDFA can be determined from the rate equations, which in general involve solving the coupled differential equations using numerical methods (Pfeiffer \& Bulow, 1992).

\section{Physical Modeling of EDFA}

Erbium is the $68^{\text {th }}$ elements of the periodic table, discovered by Carl Gustaf Mosander in 1843. It plays the main role in EDFA amplifier.
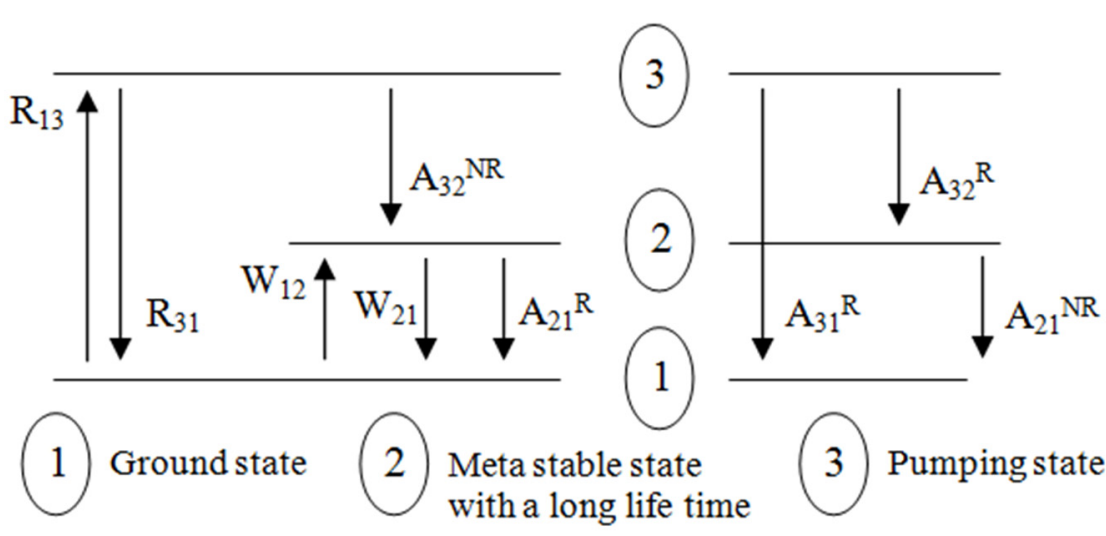

Figure 2. Three level model of an EDFA

The energy level diagram of Er-ion is shown in Figure 2 (Agrawal, 2005). When pumped at 980-nm the EDFA acts as a three level system. In the active fiber, let us assume that signal intensity and the pump signal intensity are separable, then we can write $\mathrm{I}_{\mathrm{S}}$ and $\mathrm{I}_{\mathrm{P}}$ as

$$
\begin{aligned}
& \mathrm{I}_{\mathrm{P}}(\mathrm{r}, \phi, \mathrm{z})=\mathrm{f}_{\mathrm{P}}(\mathrm{r}, \Phi) \mathrm{I}_{\mathrm{P}}(\mathrm{z}) \\
& \mathrm{I}_{\mathrm{S}}(\mathrm{r}, \phi, \mathrm{z})=\mathrm{f}_{\mathrm{S}}(\mathrm{r}, \Phi) \mathrm{I}_{\mathrm{S}}(\mathrm{z})
\end{aligned}
$$

here $f_{P}$ and $f_{S}$ are the intensity profiles of pump and signal transverse, respectively, normalized as

$$
\int_{0}^{2 x} d \phi \int_{0}^{\infty} f_{P, S}(r, \phi) r d r=1
$$

Similarly, the population density is

$$
N_{i}(r, \phi, z)=g(r, \phi) N_{0}(z)
$$


Here the total $\mathrm{Er}^{3+}$-ion doping concentration is $\mathrm{N}_{0}$ and the normalized doping intensity profile is $\mathrm{g}(r, \Phi)$. In the fiber core, if doping of $\mathrm{Er}^{3+}$ is uniform, then $\mathrm{N}_{0}(z)$ equals $\mathrm{N}_{0}$.

The overlapping integral factor, between fields and dopants, $\Gamma$ in the semiconductor laser context is

$$
\Gamma_{P, S}=\int_{0}^{2 \pi} d \phi \int_{0}^{a} f_{P, S}(r, \phi) g(r, \phi) r d r
$$

here $\mathrm{Er}^{3+}$-ion doping radius is $a$.

The effects of absorption, stimulated emission and spontaneous emission on the populations of the ground and meta-stable levels are governed by the rate equation. For EDFA in an $(r, \phi, z)$ - cylindrical coordinate system where $z$ axis as the fiber axis, the rate equation is

$$
\begin{gathered}
\frac{d N_{2}(r, \phi, z, t)}{d t}=\frac{\sigma_{a p} I_{p}(r, \phi, z)}{h v_{p}} N_{1}(r, \phi, z, t)+\frac{\sigma_{a s} I_{s}(r, \phi, z)}{h v_{s}} N_{1}(r, \phi, z, t) \\
-\frac{\sigma_{e s} I_{s}(r, \phi, z)}{h v_{s}} N_{2}(r, \phi, z, t)-A_{21} N_{2}(r, \phi, z, t) \\
N_{1}(r, \phi, z, t)+N_{2}(r, \phi, z, t)=N_{0}(r, \phi, z, t)
\end{gathered}
$$

here $\mathrm{N}_{1}$ is the ground level population density and $\mathrm{N}_{2}$ is the meta stable level population density, $v_{\mathrm{p}}$ is pump frequency and $v_{\mathrm{s}}$ is signal frequency, $h$ is Plank's constant, $\sigma_{\mathrm{as}}$ is the stimulated absorption and $\sigma_{\mathrm{es}}$ is the stimulated emission cross sections between the ground and the meta stable state; the absorption cross section from the ground state to the pump level is $\sigma_{\text {ap }}$ and $\mathrm{A}_{21}\left(=1 / \tau_{21}\right)$ is the spontaneous emission rate.

If the pump light and signal light travel in the same direction along z-coordinate and the fiber loss are neglected, then the beams propagation equations can be written as:

$$
\begin{gathered}
\frac{d I_{p}(r, \phi, z)}{d z}=-\sigma_{a p} N_{1}(r, \phi, z, t) I_{p}(r, \phi, z) \\
\frac{d I_{s}(r, \phi, z)}{d z}=\left[\sigma_{e s} N_{2}(r, \phi, z, t)-\sigma_{a s} N_{1}(r, \phi, z, t)\right] I_{s}(r, \phi, z)
\end{gathered}
$$

If the time derivative in equation (6) set to be zero, then the problem reduced to the steady state case. Afterward, if we integrate equations (6) to (9) across the fiber transverse plane, we get -

$$
\begin{gathered}
0=\frac{d N_{2}(z, t)}{d t}=\frac{\sigma_{a p} \Gamma_{p} P_{p}(z)}{A_{c} h v_{p}} N_{1}(z, t)+\frac{\sigma_{a s} \Gamma_{s} P_{s}(z)}{A_{c} h v_{s}} N_{1}(z, t) \\
-\frac{\sigma_{e s} \Gamma_{s} P_{s}(z)}{A_{c} h v_{s}} N_{2}(z, t)-A_{21} N_{2}(z, t) \\
N_{1}(z)+N_{2}(z)=N_{0} \\
\frac{d P_{p}(z)}{d z}=-\Gamma_{p} \sigma_{a p} N_{1}(z) P_{p}(z) \\
\frac{d P_{s}(z)}{d z}=\Gamma_{p}\left[\sigma_{e s} N_{2}(z)-\sigma_{a s} N_{1}(z)\right] P_{s}(z)
\end{gathered}
$$

here $\operatorname{Pp}(\mathrm{z})$ is the pump power and $\operatorname{Ps}(\mathrm{z})$ is the signal power at position $\mathrm{z}$ in the fiber; the efficient core area is denoted by $\mathrm{A}_{\mathrm{c}}$ for $\mathrm{Er}^{3+}$-doped fiber. We can rewrite the equation (10) as,

$$
N_{2}(z)=\frac{\tau \Gamma_{p} \sigma_{a p} P_{p}(z)}{A_{c} h v_{p}} N_{1}(z)+\frac{\tau \Gamma_{s}\left[\sigma_{a s} N_{1}(z)-\sigma_{e s} N_{2}(z)\right] P_{s}(z)}{A_{c} h v_{s}}
$$

where $\tau$ equal to $\tau_{21}$. Then, Eqn. (12) $\times \frac{\tau}{A_{c} h v_{p}}+$ Eqn. (13) $\times \frac{\tau}{A_{c} h v_{s}}+$ Eqn. (14) has been made and the obtained result is as follows, 


$$
N_{2}+\frac{\tau}{h v_{p}} \frac{d P_{p}(z)}{d z}+\frac{\tau}{h v_{s}} \frac{d P_{s}(z)}{d z}=0
$$

Then equations (12)-(15) are integrated over space $\mathrm{z}$ and it has been assumed that, $\mathrm{S}=\int_{0}^{z} N 2(z) d z$.
After careful calculations it has been found that,

$$
\begin{aligned}
S & =\frac{\ln \frac{P_{P}(z)}{P_{P}(0)}+\Gamma_{P} \sigma_{a p} N_{0} z}{\Gamma_{P} \sigma_{a p}}=\frac{\ln \frac{P_{S}(z)}{P_{S}(0)}+\Gamma_{S} \sigma_{a s} N_{0} z}{\Gamma_{S}\left(\sigma_{a s}+\sigma_{e s}\right)} \\
& =-\frac{\tau}{A_{c} h v_{P}}\left[P_{P}(z)-P_{P}(0)\right]-\frac{\tau}{A_{c} h v_{S}}\left[P_{S}(z)-P_{S}(0)\right]
\end{aligned}
$$

The amplification is defined as $\mathrm{G}(\mathrm{z})=P_{\mathrm{s}}(\mathrm{z}) / P_{\mathrm{s}}(0)$. Now from equation (16)

$$
[G(z)]^{\beta} \exp \left(-\beta \Gamma_{S} \sigma_{e s} N_{0} z\right)=1-\frac{v_{P} P_{S}(0)}{v_{S} P_{P}(0)}[G(z)-1]-\frac{\left[\ln G(z)+\Gamma_{S} \sigma_{a s} N_{0} z\right] A_{c} h v_{P}}{\tau P_{P}(0) \Gamma_{S}\left(\sigma_{a s}+\sigma_{e s}\right)}
$$

here, $\beta=\frac{\Gamma_{p} \sigma_{a p}}{\Gamma_{s}\left(\sigma_{a s}+\sigma_{e s}\right)}$

This equation is an indirect analytical solution for the amplifier gain.

For the pump threshold power $G(L)=1$, which means that at this pump power the signal remains unchanged after traveling the fiber. Substituting it in equation (17), we have

$$
P_{p t h}=\frac{A_{c} h v_{P} \sigma_{a s} N_{0} L}{\tau\left(\sigma_{a s}+\sigma_{e s}\right)\left[1-\exp \left(-\beta \Gamma_{S} \sigma_{e s} N_{0} L\right]\right.},
$$

where the fiber length is $L$.

\section{Simulation and Results}

Several C programs have been written to solve the equations and the result data are manipulated in MATLAB to represent the results.

Figure 3 and Figure 4 show the variation of amplifier gain with pump power and fiber length. Figure 3 is for the large signal launching power $(1 \mathrm{~mW})$, while Figure 4 is for the small signal launching power $(1 \mu \mathrm{W})$.

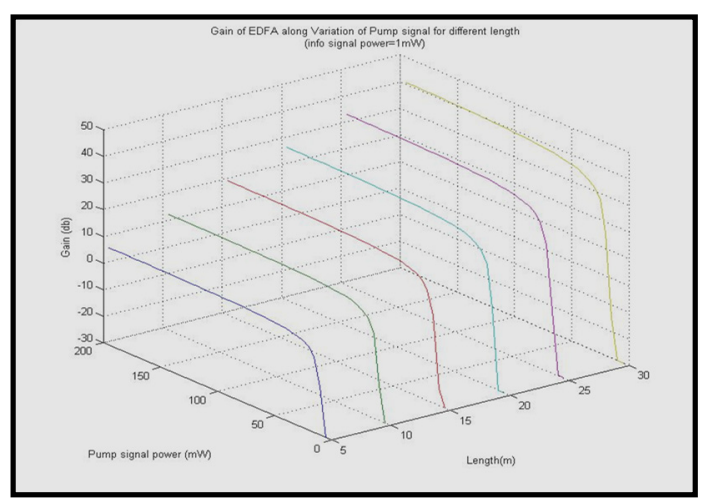

Figure 3. Gain of EDFA on pump power and fiber length for large signal power

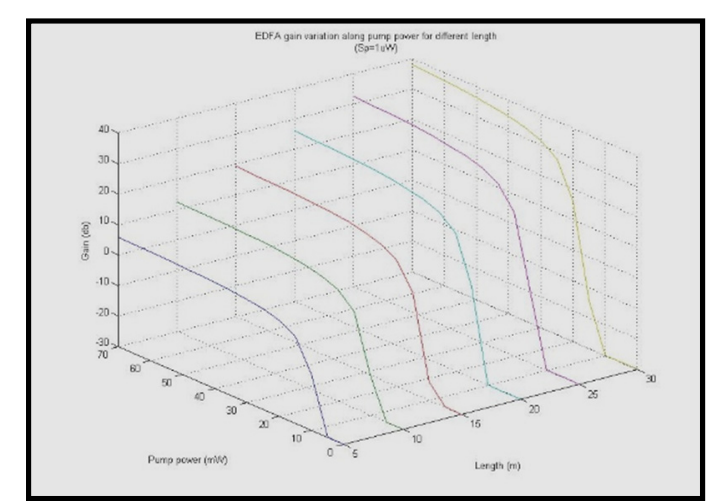

Figure 4. Gain of EDFA on pump power and fiber length for small signal power

The amplifier gain increases with the increase of pump power and become saturated at high pump power. The smaller the length of the fiber amplifier, the lower the pump power at which the gain saturation occurs. This is because in a shorter fiber, there are less $\mathrm{Er}^{3+}$-ions, thus total population inversion is obtained at lower pump power and the gain saturation occurs more easily. The gain under large signal launching power is evidently lower than that of the small signal launching power for the same parameters.

The amplifier gain along with the fiber length versus pump power are illustrated in the Figure 5 and Figure 6. Figure 5 is for the large signal launching power $(1 \mathrm{~mW})$, while Figure 6 is for the small signal launching power $(1 \mu \mathrm{W})$. 


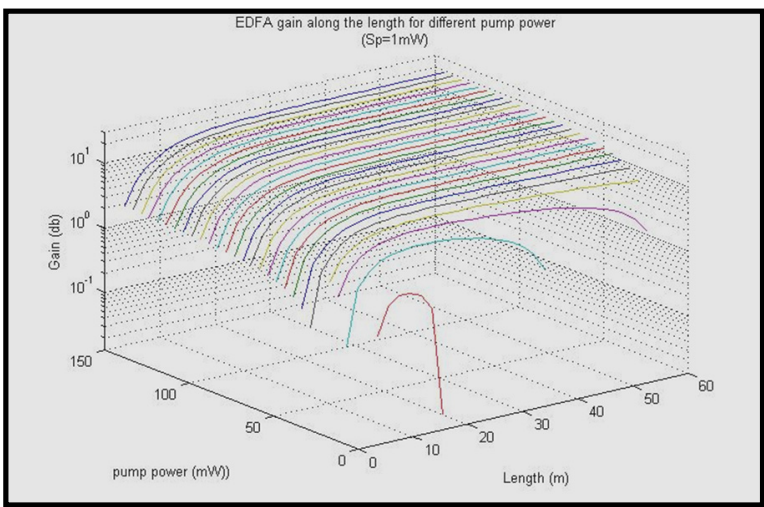

Figure 5. Variation of gain along with the fiber for different pump power for large signal power

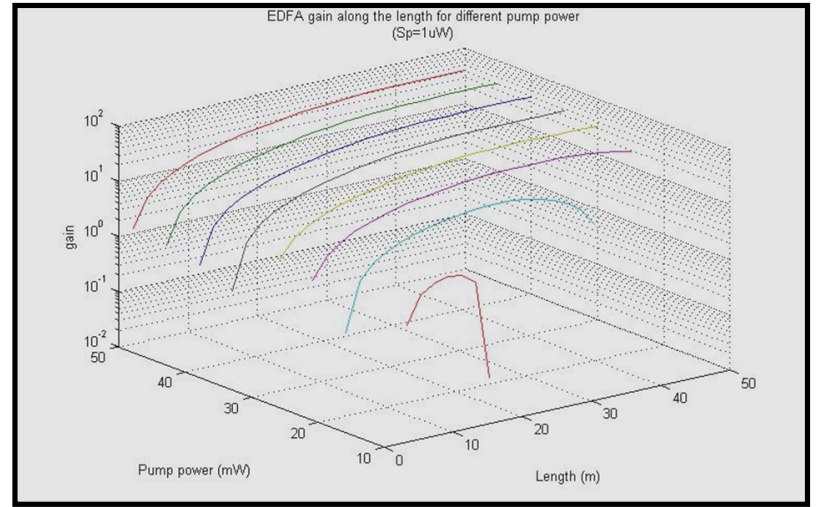

Figure 6. Variation of gain along the fiber for different pump power for small signal power

As shown in the figures, the gain increases to the maximum value, and then decreases along with the fiber length, which means that the gain has a nonlinear relation to the fiber length. The fiber length for which the gain is maximum, is the optimum amplifier length. So that, from here optimum fiber length can be found for various sets of other parameters combination.

Figure 7 and Figure 8 show the amplifier gain along with the fiber length versus $\mathrm{Er}^{3+}$ ion doping concentration for constant pump power. Figure 7 is for the large signal power $(1 \mathrm{~mW})$, while Figure 8 is for the small signal launching power $(100 \mu \mathrm{W})$. The amplifier gain increases with the increase of pump power and become saturated at longer length. This graph depicts a very important property that, higher the $\mathrm{Er}^{3+}$-ion concentration, the shorter the optimum amplifier length required at which the gain reaches maximum.

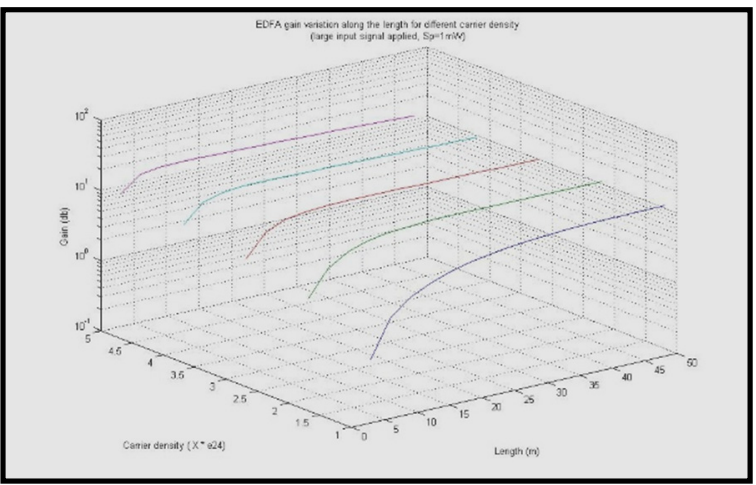

Figure 7. Variation of gain along the fiber for different carrier concentration for large signal power

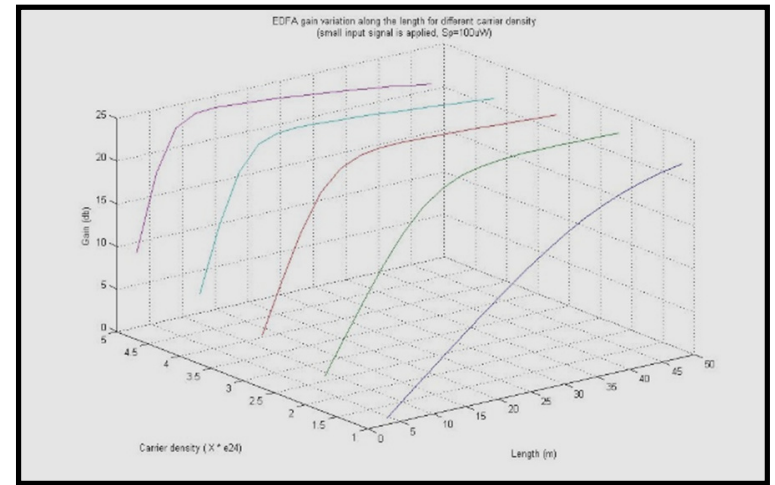

Figure 8. Variation of gain along the fiber for different carrier concentration for small signal power

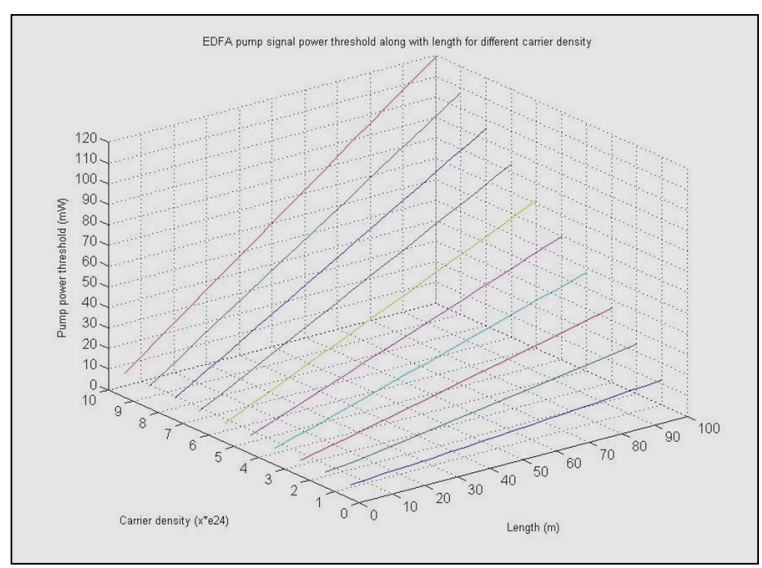

Figure 9. The pump threshold power for the different combination of length and carrier density 
Figure 9 shows the pump threshold power increases linearly with the length of the fiber amplifier as well as with the carrier density. This is because when the fiber becomes longer the signal absorption becomes stronger.

\section{Conclusion}

A lot of works have been done to improve the performances of EDFA since it was born. Based on rate and propagation equations, a series of equations have been derived to analyze the characteristics of EDFA pumped at $980-\mathrm{nm}$. In the steady state, analytical solutions for amplifier gain, optimum length and pump threshold are obtained. The gain of the EDFA can be maximized by using appropriate design parameters and appropriate value of the material properties. Results show that for a given pump power there exists an optimum amplifier length for maximum gain. Also the results can be used for the optimum design of erbium-doped fiber amplifier.

\section{Reference}

Agrawal, G. P. (2005). Fiber-optic communication Systems (3rd ed.). John Wiley and Sons.

Gerd, K. (2000). Optical fiber communications. McGraw-Hill Higher Education.

Khare, R. P. (2004). Fiber optics and optoelectronics. Oxford University Press.

Kreyszig, E. (1993). Advanced Engineering Mathematics (7th ed.). John Wiley and Sons.

Mears, R. J., Reekie, L., Jauncey, M., \& Payne, D. N. (1987). Low-noise erbium-doped fiber amplifier operating at $1.54 \mu \mathrm{m}$. Electronics Letter, 26, 1026.

Pfeiffer, T., \& Bulow H. (1992). Analytical gain equation for erbium-doped fiber amplifiers including mode field profiles and dopant distribution. Photonics Technology Letters, 4(5), 449-451.

Saleh, A. A., Jopson, R. M., Evankow, J. D., \& Aspell J. (1990). Modeling of Gain Erbium-doped fiber amplifier. IEEE Photonic Technology letters, 2, 714-717.

\section{Copyrights}

Copyright for this article is retained by the author(s), with first publication rights granted to the journal.

This is an open-access article distributed under the terms and conditions of the Creative Commons Attribution license (http://creativecommons.org/licenses/by/3.0/). 